\title{
Probing the Influence of X-rays on Aqueous Copper Solutions Using Time-Resolved in Situ Combined Video/X-ray Absorption Near-Edge/Ultraviolet-Visible Spectroscopy
}

\author{
J. Gerbrand Mesu, Andrew M. Beale, Frank M. F. de Groot, and Bert M. Weckhuysen* \\ Inorganic Chemistry and Catalysis, Department of Chemistry, Utrecht University, Sorbonnelaan 16, \\ 3584 CA Utrecht, The Netherlands
}

Received: April 28, 2006; In Final Form: July 12, 2006

\begin{abstract}
Time-resolved in situ video monitoring and ultraviolet-visible spectroscopy in combination with X-ray absorption near-edge spectroscopy (XANES) have been used for the first time in a combined manner to study the effect of synchrotron radiation on a series of homogeneous aqueous copper solutions in a microreactor. This series included both non biologically relevant (pyridine, bipyridine, neocuproine, terpyridine, dimethylpyridine, ammonia, ethylenediamine, and 1,10-phenanthroline) and biologically relevant (histidine, glycine, and imidazole) ligands. It was found that when water is present as solvent, gas bubbles are formed under the influence of the X-ray beam. At the liquid-gas interface of these bubbles, in particular cases colloidal copper nanoparticles are formed. This reduction process was found to be influenced by the type of copper precursor salt $\left(\mathrm{SO}_{4}{ }^{2-}, \mathrm{NO}_{3}{ }^{-}\right.$, and $\left.\mathrm{Cl}^{-}\right)$, the ligands surrounding the copper cation, and the redox potential of the copper complexes (ranging between +594 and $-360 \mathrm{mV}$ ). In other words, in some cases, no reduction was encountered (e.g., ammonia in the presence of $\mathrm{SO}_{4}{ }^{2-}$ and $\mathrm{NO}_{3}{ }^{-}$), whereas in other cases reduction to either $\mathrm{Cu}^{+}$(neocuproine with $\mathrm{SO}_{4}{ }^{2-}$ ) or $\mathrm{Cu}^{0}$ (e.g., histidine and imidazole both with $\mathrm{SO}_{4}{ }^{2-}, \mathrm{NO}_{3}{ }^{-}$, and $\mathrm{Cl}^{-}$) was observed. These results illustrate the added value of video spectroscopy for the interpretation of in situ XANES studies. Not only do the results give an illustration of the parameters that are important in the redox processes that occur in biological systems, they also show the potential problems associated with studying catalytic processes in aqueous solutions by XANES spectroscopy.
\end{abstract}

\section{Introduction}

X-ray absorption near-edge spectroscopy (XANES) is an element-specific characterization technique that is commonly applied in various fields of chemistry for the elucidation of the oxidation state and coordination environment of transition metals. To obtain this kind of information, the sample has to be exposed to X-rays. XANES is, in a way, a destructive characterization technique since a photoelectron is ejected from one of the core levels of the absorber atom, leaving behind a core hole., ${ }^{1,2}$ Via different kinds of radiative and nonradiative decay processes, the core hole in the absorber atom is filled by electrons from higher shells. For all shells other than 1s, Auger is the dominant decay channel, so the most likely path is a $1 \mathrm{~s} 2 \mathrm{p}$ radiative decay process, followed by various Auger channels. This ultimately leaves an absorber atom with two, three, and possibly more electrons missing in the shell with the highest energy, the valence shell. Most of the time, the holes are filled up by electrons from the direct environment of the absorber atom, and in that way the direct surroundings of the absorber atom are affected. If this is not the case, charging of the specimen may occur.

When dealing with X-rays, one can generally distinguish between two types of radiation damage. Primary beam damage is caused by the direct interaction of the ionizing radiation with the electrons in the object via photoelectric, Auger, or Compton effects, which can cause direct damage to the chemical bonds within the specimen. This interaction also leads to the formation of energetic electrons, which are capable of generating additional

* To whom correspondence should be addressed. Phone: +31-302534328. Fax: +31-30-2511027. E-mail: b.m.weckhuysen@chem.uu.nl. reactive species such as free radicals, which can also react with and alter the chemical state of the specimen. This is known as secondary beam damage. For crystalline samples a third type of radiation damage may also occur when the initial primary or secondary beam damage can cause a destabilization of the lattice, leading it to collapse, a process often referred to as tertiary beam damage. ${ }^{3,4}$ The primary beam damage only depends on the energy of the incident radiation and the photons absorbed, while secondary and tertiary beam damages depend on the properties of the specimen.

With the advent of high-brilliance, highly focused X-ray beams from third-generation synchrotron sources, the observation of beam damage or beam influence in various types of samples is becoming more common and poses a number of problems. For example, it becomes difficult to determine the physical and chemical properties of the sample such as its structure or function. ${ }^{5-12}$ This is especially important for the study of catalytic mechanisms since specific species, often considered as potential reaction intermediates and active sites, are formed in a reaction mixture. Normally such information is achieved under time-resolved measurement conditions, but one should be aware that the X-ray beam itself might induce the observed changes to the catalytic system under study, especially in circumstances where the experimental time resolution relies on increasing photon statistics rather than improvements in detector performance. ${ }^{13}$ While a number of novel approaches have been developed to counter this problem, it is clear that to fully utilize the X-ray beams now available a more fundamental understanding of the chemical processes involved in radiation damage is of paramount importance, so, where possible, 
SCHEME 1: Ligands Used in the Copper Complexes That Were Tested in Aqueous Solutions under the Influence of the X-ray Beam: (a) Pyridine, (b) Imidazole, (c) Bipyridine, (d) L-Histidine, (e) Neocuproine, (f) Terpyridine, (g) Glycine, (h) Dimethylpyridine, (i) Ammonia, (j) 1,10-Phenanthroline, (k) Ethylenediamine
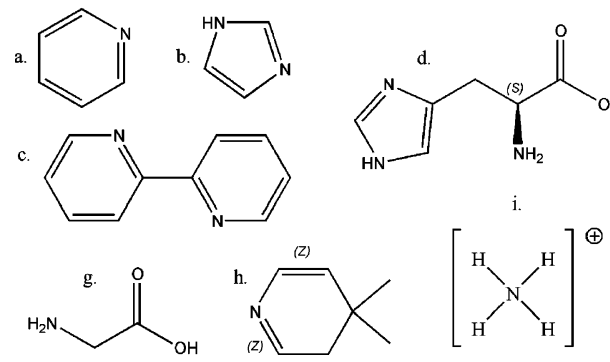

appropriate actions can be taken. ${ }^{14-17}$ However, it should also be noted that this effect is not always considered with such a negative perspective since it has also been successfully utilized in a novel way to prepare nanometer-sized metallic clusters. ${ }^{18-20}$

This work was originally targeted toward kinetic studies on the catalytic mechanism of aqueous $\mathrm{Cu}^{2+}$-containing solutions by using a combined in situ ultraviolet-visible (UV-vis)/ XANES setup. However, the X-rays caused problems with the reproducibility of the kinetic measurements and led finally to the observation that the X-rays themselves can have a strong effect on the catalytic activity and stability of the copper complexes under study. In this paper, we present a systematic study on the influence of the effect of the X-ray beam on aqueous solutions containing copper complexes. Ligands which are both non biologically relevant (pyridine, bipyridine, neocuproine, terpyridine, dimethylpyridine, ammonia, ethylenediamine, and 1,10-phenanthroline) and biologically relevant (histidine, glycine, and imidazole) are investigated. Next to in situ UV-vis spectroscopy and XANES, high-resolution video spectroscopy was applied to obtain a clearer insight into the chemical processes that are occurring within the cuvette of the stopped-flow microreactor as soon as the aqueous copper solutions are exposed to the X-ray beam. An additional advantage to the video imaging is that it allowed us to probe the contents of the entire cuvette rather than simply the area at the focal point of the incoming light, which is typically at a maximum for UV-vis light on the order of a few millimeters and is therefore comparatively smaller. This allowed us to observe more clearly the processes of $\mathrm{Cu}^{2+}$ reduction and colloidal particle formation. We believe that this study is the first of its kind where the use of a combination of real timeresolved in situ video monitoring and UV-vis spectroscopy has been used to give detailed insight into the potential effects that $\mathrm{X}$-ray beams may have on aqueous transition-metal-ion solutions. Furthermore, the observed findings may have broader implications for the field of transition-metal-ion catalysis as well as bioinorganic chemistry, as it gives an illustration of the factors that are important in the redox processes that occur in biological systems.

\section{Experimental Section}

Chemicals and Solutions. An overview of the different ligands of which aqueous copper complexes were prepared is shown in Scheme 1. The metal salts $\mathrm{Cu}\left(\mathrm{NO}_{3}\right)_{2} \cdot 3 \mathrm{H}_{2} \mathrm{O}$ (Acros Organics, p.a.), $\mathrm{CuSO}_{4} \cdot 5 \mathrm{H}_{2} \mathrm{O}$ (Merck, p.a.), and $\mathrm{CuCl}_{2}$ (Merck, anhydrous $99 \%$ ), the ligands pyridine (Acros Organics, 99+\%), imidazole (Acros Organics, 99+\%), L-histidine (Janssen Chimica, 98\%), neocuproine (Sigma-Aldrich), 2,2':6',2"'-terpyridine (Aldrich, 98\%), glycine (Acros Organics, p.a.), ammonia (Merck, 25\% solution, p.a.), and ethylenediamine (Acros Organics, 99\%), and the solvents water (99\%) and NMP (1-

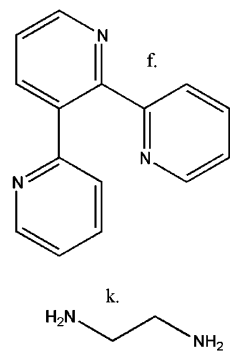

methyl-2-pyrrolidinone; Biosolve, HPLC grade) were all used without further purification. The concentrations, the ligand to copper ratios, and the copper precursor salts used for the preparation of the solutions are summarized in Table S1 in the Supporting Information.

Spectroscopy. The video/UV-vis/ED-XANES data were collected at the undulator beamline ID24 at the European Synchrotron Radiation Facility (ESRF) (Grenoble, France). A specially designed setup was used (see Figure 1 for a schematic overview) which was based on a setup previously used to obtain combined UV-vis/ED-XANES data. ${ }^{21}$ In this setup either video movies or UV-vis spectra can be collected in combination with the ED-XANES data. The video movies and the UV-vis spectra are collected in an orientation perpendicular to the direction of the X-ray beam.

For measurements, the solutions were brought into a specially designed black quartz cuvette using the commercially available Bio-logic stopped-flow module (SFM-400). The cuvette has a path length in both the X-ray and UV-vis directions of $5 \mathrm{~mm}$ and a total sample volume of $80 \mu \mathrm{L}$. The windows in the X-ray direction are made out of vitreous carbon (1 mm thick) to minimize X-ray absorption, whereas the windows perpendicular to the X-rays through which the UV-vis and video data were collected are made of clear quartz. An approximate X-ray photon

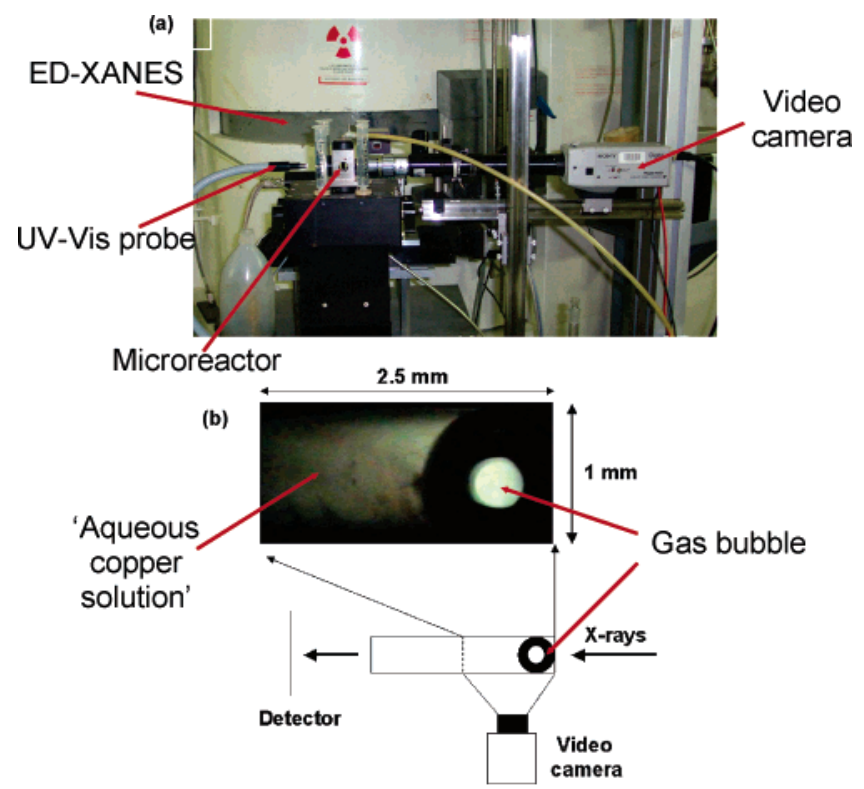

Figure 1. (a) Overview picture of the video camera focused on the cuvette in the observation head on top of the stopped-flow module in the experimental hutch of beamline ID24 at the ESRF. (b) Enlarged view of the video image obtained through the cuvette window and schematic of the experimental setup detailing where the video camera image was taken in relation to the cuvette and incoming X-ray beam. 


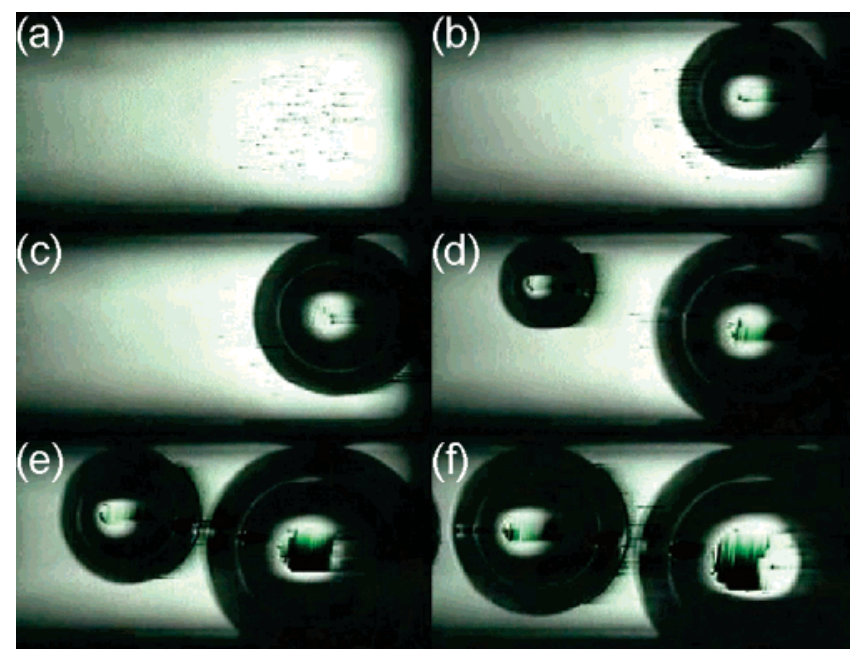

Figure 2. Demineralized water exposed to X-rays as a function of time, as probed by video spectroscopy after (a) $0 \mathrm{~s}$, (b) $400 \mathrm{~s}$, (c) 500 $\mathrm{s}$, (d) $750 \mathrm{~s}$, (e) $1000 \mathrm{~s}$, and (f) $1200 \mathrm{~s}$.

flux of $10^{12}$ photons $\mathrm{s}^{-1}$ over an energy range of 8910-9250 $\mathrm{eV}$ was focused using a curved $\mathrm{Si}(111)$ Bragg polychromator crystal to a spot size of $0.03 \times 0.2 \mathrm{~mm}^{2}(H \times V$, fwhm). The XANES data were collected using a phosphorus-masked Peltiercooled Princeton CCD camera. The UV-vis spectra (in the range from 200 to $735 \mathrm{~nm}$ ) were collected in transmission mode, perpendicular to the incident X-ray beam, using an MMS-UV1/ 500-1 high-speed diode spectrometer equipped with optical fibers. The video movies were recorded at a speed of 25 frames $\mathrm{s}^{-1}$ using a Sony Digital Hyper HAD color video camera, equipped with a high-magnification lens (see Figure 1a).

\section{Results and Discussion}

Radiolysis of Aqueous Solutions. First, as a blank experiment, demineralized water (without copper) was exposed to the $\mathrm{X}$-ray beam. As soon as the sample was exposed to the X-ray beam, the formation of gas bubbles in the cuvette was observed. Snapshots from the in situ video movie at different moments in time are presented in Figure 2. As can be seen in Figure 2, gas bubbles are formed which grow in time, so after $1200 \mathrm{~s}$ the gas bubbles almost occupy the entire cell volume. The experiment was repeated several times, and every time the formation of bubbles was observed. The speed of the growth of the bubbles was found to be dependent on the flux of the incoming X-ray beam: the higher the flux, the faster the speed of growth of the gas bubbles. This observation strongly indicates that the gas is formed as a result of a direct interaction between the X-rays and the water. In the literature this process is known as radiolysis; which is the disintegration of molecular species under the influence of radiation. ${ }^{22,23}$ Radiolysis of water is known to cause fragmentation of the water to form a number of both radical and ionic species. This fragmentation phenomenon is both a primary and a secondary process since the high-energy beam first produces a "free electron" which is able to undergo further reaction with water as demonstrated in eq $1.24,25$

$$
\mathrm{H}_{2} \mathrm{O}+h v_{\mathrm{X}-\text { ray }} \rightarrow \mathrm{H}_{2} \mathrm{O}^{+}+\mathrm{e}_{\text {dry }}^{-} \stackrel{\mathrm{H}_{2} \mathrm{O}}{\longrightarrow} \mathrm{H}_{3} \mathrm{O}^{+}+\mathrm{HO}^{\bullet}+\mathrm{e}^{-} \text {aq }
$$

The majority of these species recombine to re-form water, but the radiolysis products individually also may recombine, forming new species, such as $\mathrm{H}_{2}$ and $\mathrm{H}_{2} \mathrm{O}_{2} \cdot{ }^{22,23,25-27}$ In high-purity water $\mathrm{H}_{2} \mathrm{O}_{2}$ is stable and accumulates, whereas the same holds for

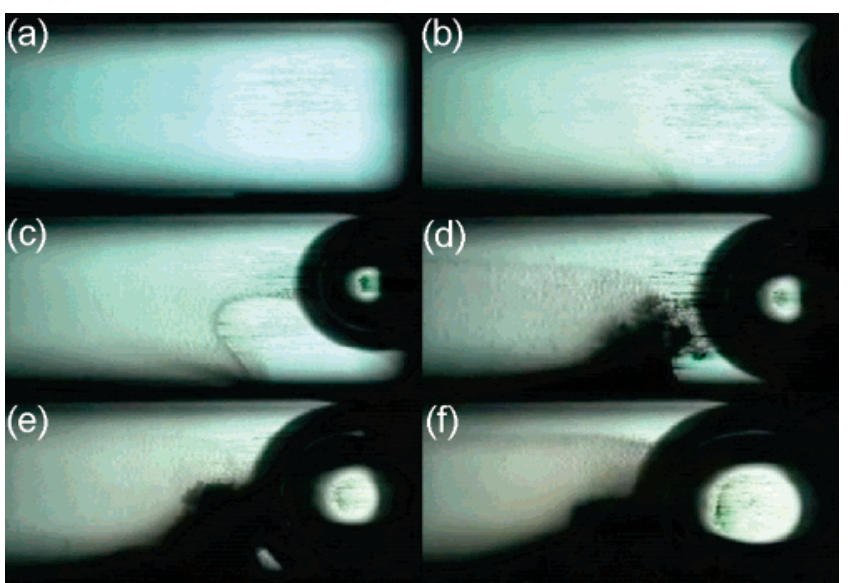

Figure 3. Aqueous $100 \mathrm{mM}$ copper-histidine (1:2) solution at $\mathrm{pH}$ 3.4 exposed to $\mathrm{X}$-rays as a function of time, as probed by video spectroscopy after (a) $0 \mathrm{~s}$, (b) $400 \mathrm{~s}$, (c) $500 \mathrm{~s}$, (d) $750 \mathrm{~s}$, (e) $1000 \mathrm{~s}$, and (f) $1200 \mathrm{~s}$.

the $\mathrm{H}_{2}$ gas. When in the initial situation a (small) bubble is present, this bubble will grow by the transportation of hydrogen gas that is formed by the radiolysis of water toward the bubble. When initially no bubble is present, the $\mathrm{H}_{2}$ concentration within the solution will increase up to a certain critical gas concentration, after which spontaneously a macroscopic bubble will be formed. Therefore, in principle, the formation of hydrogen gas (and eventually the formation of gas bubbles) cannot be prevented when aqueous solutions are exposed to X-rays. However, the rate of the formation of the hydrogen gas will be larger for high-flux X-ray beamlines than it is for low-flux X-ray beamlines.

The situation becomes more complicated when solutes are present in the water, as these solutes can start to interfere with the recombination process of water after radiolysis or react with the accumulating $\mathrm{H}_{2}$ and $\mathrm{H}_{2} \mathrm{O}_{2}$ species. ${ }^{22,23,25-27}$ At low solute concentrations the interactions between the radiolysis products of water and the solute will prevail, but at higher solute concentrations also the products of the radiolysis of the solute and the interaction with their environment may start to play an important role. Therefore, if one wants to study the effect of the radiolysis products of water on a certain system, solute concentrations should be kept as low as possible. In our case we chose to study aqueous solutions of different copper complexes at concentrations of 100 and $20 \mathrm{mM}$, as we were interested in the catalytic mechanism of copper-complexcatalyzed oxidation reactions. ${ }^{28}$

Copper-Histidine. When an aqueous solution containing 100 $\mathrm{mM} \mathrm{Cu}{ }^{2+}$-histidine $(1: 2)$ complex at $\mathrm{pH} 3.4$ was exposed to the X-ray beam, again the formation of gas bubbles was observed. However, this time additional phenomena could be detected. This is shown in Figure 3, where snapshots obtained from the in situ video movie (Supporting Information, Movie S1) are presented. Next to the gradual bubble formation, colloidal particles start to form, beginning at the position where the X-ray beam is entering the cuvette. This can be seen in Figure $3 \mathrm{c}, \mathrm{d}$, where a jet of particles can be observed which almost appears to come from the gas bubble itself. In time these particles pile up at the bottom of the cuvette as debris. It is difficult at this stage to be sure what is responsible for the reduction of the copper complex to form the colloidal metallic particles. The following can be considered as plausible explanations. First, the presence of $\mathrm{H}_{2}$ in the solution (Figure $3 \mathrm{~b}$ ) and in the gas bubble (Figure 3c) reacts to reduce the $\mathrm{Cu}^{2+}$ in the aqueous solution to $\mathrm{Cu}^{0}$, leading to the formation of colloidal 


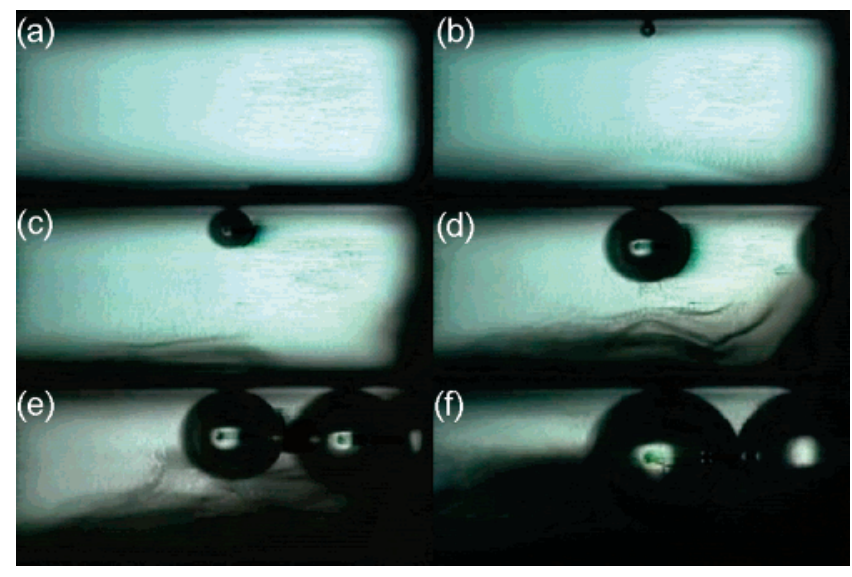

Figure 4. Aqueous copper-histidine (1:2) solution at $\mathrm{pH} 6.0$ exposed to X-rays as a function of time, as probed by video spectroscopy after (a) $0 \mathrm{~s}$, (b) $45 \mathrm{~s}$, (c) $120 \mathrm{~s}$, (d) $240 \mathrm{~s}$, (e) $360 \mathrm{~s}$, and (f) $600 \mathrm{~s}$.

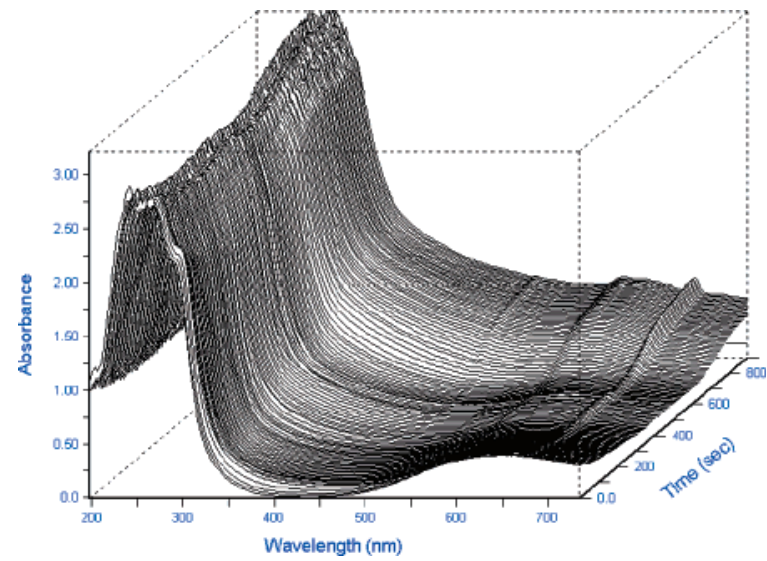

Figure 5. UV-vis spectra of the $100 \mathrm{mM}$ aqueous copper-histidine $(1: 2)$ solution at $\mathrm{pH} 6.0$ as a function of the exposure time to the X-ray beam.

copper particles. Alternatively, a reaction with the "hydrated electron", which is also one of the products of the radiolysis of water (eq 1), may lead to a direct reduction.

In the next experiment again an aqueous solution containing $100 \mathrm{mM} \mathrm{Cu}^{2+}$-histidine (1:2) complex, but this time at $\mathrm{pH}$ 6.0, was exposed to the $\mathrm{X}$-ray beam. The $\mathrm{pH}$ of this solution was adjusted to 6.0 using a dilute $\mathrm{NaOH}$ solution. Next to video monitoring, also in situ UV-vis and XANES spectra were collected on the same sample. The data are presented in Figures 4-6. Figure 4 shows the different snapshots from the in situ video movie (Supporting Information, Movie S2). Again the same phenomena as with the copper-histidine solution at $\mathrm{pH}$ 3.4 can be observed: gas bubbles and colloidal copper particles are formed in time. However, the formation of the colloidal copper particles occurs this time on a much faster time scale. After only $45 \mathrm{~s}$ (Figure 4b) a wave of colloidal particles comes down from the plane of the X-ray beam (which is focused in the center of the cuvette). This process is repeated several times within the first few minutes of the solution being exposed to the X-ray beam. The different layers from the several waves of colloidal particles gradually pile up (due to gravity) at the bottom of the cuvette as can be seen in Figure 4c,d. From these results it is strongly suggested that the colloidal copper particles are formed along the plane of the X-ray beam and thus that the $\mathrm{X}$-ray beam is responsible for the reduction of the copper. It is not clear whether this is due to primary or secondary damage.

Additional evidence for $\mathrm{Cu}^{2+}$ reduction was observed in the $\mathrm{UV}-$ vis and XANES data. Figure 5 shows the UV-vis spectra

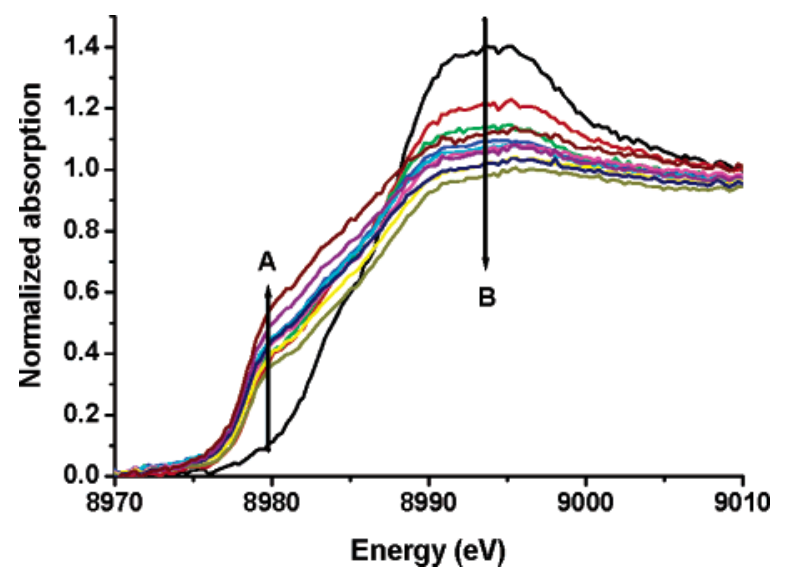

Figure 6. XANES spectra of the aqueous $\mathrm{Cu}$-histidine (1:2) solution at $\mathrm{pH} 6$ as a function of time. Arrows $\mathrm{A}$ and $\mathrm{B}$ indicate the trend as a function of the reaction time. The time interval between two successive XANES spectra is $20 \mathrm{~s}$.

of the content of the cuvette as a function of the exposure time to the $\mathrm{X}$-ray beam. Initially, the $\mathrm{d}-\mathrm{d}$ transition of the $\mathrm{Cu}(\mathrm{II})-$ histidine (1:2) complex at $650 \mathrm{~nm}$ can clearly be observed. ${ }^{29,30}$ The intensity of this absorption band decreases with exposure time, which is indicative of the disappearance of $\mathrm{Cu}^{2+}$ and appearance of either $\mathrm{Cu}^{+}$or $\mathrm{Cu}^{0}$. No new absorption bands appeared in the region 400-600 $\mathrm{nm}$, which can be attributed to metal-to-ligand charge transfer (MLCT) bands, nanostructured $\mathrm{Cu}_{2} \mathrm{O}$, or metallic copper. ${ }^{31-33}$ However, after about $400 \mathrm{~s}$ the overall background of the UV-vis spectra starts to increase with time. This can be explained either by an increase in scattering of the UV-vis light due to the presence of colloidal copper particles or by the effect of the gas bubble in the optical path of the spectrometer (which might also cause scattering or allow greater transmission). Further evidence comes from the in situ, time-resolved XANES spectra (Figure 6) that are collected with the same X-ray beam that induces the formation of the colloidal copper particles. Two phenomena can be observed, which support the observation that the copper within the plane of the X-ray beam is being reduced: a pre-edge feature at $8980 \mathrm{eV}$ (indicated by arrow A in Figure 6) is observed to form and increase, and the intensity of the white line (indicated by arrow $\mathrm{B}$ in Figure 6) decreases. The two observations are consistent with the reduction of $\mathrm{Cu}^{2+}$ to $\mathrm{Cu}^{0}{ }^{13}$ In addition, the features that are present in the EXANES region of the absorption spectrum (not shown) start to resemble the features that also can be found in the EXANES region of the X-ray absorption spectrum of a copper foil.

Copper-Neocuproine. To further investigate the reducing influence of the X-ray beam, we have studied a solution containing the $\mathrm{Cu}^{2+}$-neocuproine complex. As the solubility of this complex is not very high in water, we used a water/ NMP (1:1) mixture as a solvent. From this complex it is known that the $\mathrm{Cu}^{+}$version has a very characteristic, strong red color, caused by MLCT transitions in the $\mathrm{Cu}^{+}$complex. ${ }^{31}$ Indeed a red coloring of the solution was observed when the $\mathrm{Cu}^{2+}$. neocuproine (1:2) complex in water/NMP (1:1) was exposed to the X-ray beam, indicating the formation of the $\mathrm{Cu}^{+}$complex under the influence of the X-ray beam. The speed at which the $\mathrm{X}$-ray beam reduces the $\mathrm{Cu}^{2+}-$ neocuproine complex is illustrated in the snapshots that are presented in Figure 7 (Supporting Information, Movie S3). As can be seen in Figure $7 \mathrm{~b}$, already within the first few seconds after the exposure of the solution to the $\mathrm{X}$-ray beam, the red color of the $\mathrm{Cu}^{+}$complex can be observed. The formation of colloidal copper formation 


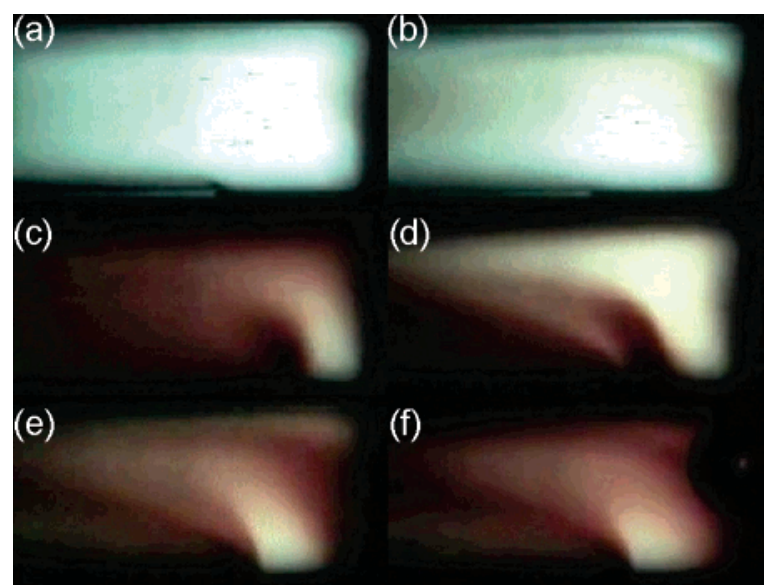

Figure 7. Copper-neocuproine (1:2) solution in water/NMP (1:1) exposed to X-rays as a function of time, as probed by video spectroscopy after (a) $0 \mathrm{~s}$, (b) $6 \mathrm{~s}$, (c) $120 \mathrm{~s}$, (d) $200 \mathrm{~s}$, (e) $400 \mathrm{~s}$, and (f) $600 \mathrm{~s}$.

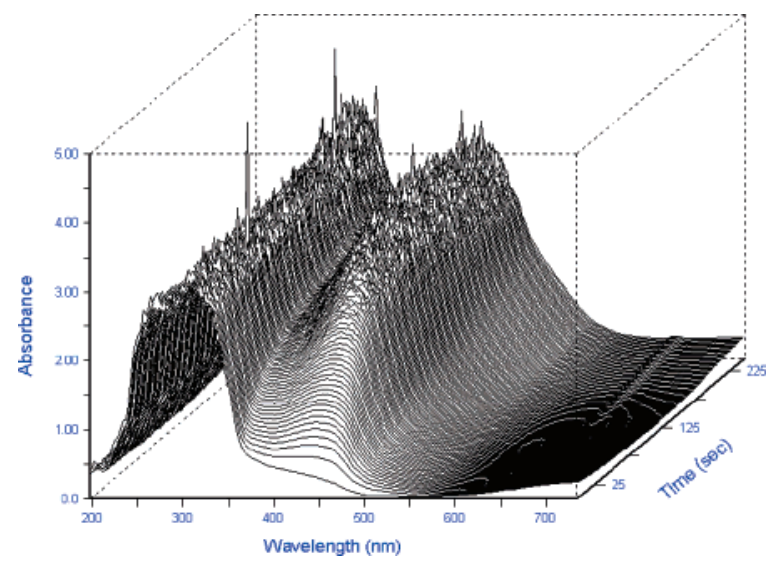

Figure 8. UV-vis spectra of the copper-neocuproine solution as a function of the reaction time.

was not observed, and the formation of gas bubbles was found to proceed much slower. This last observation is understandable, as less water is present within the cuvette and thus the radiolysis process of water is suppressed. Additional evidence for the formation of the $\mathrm{Cu}^{+}$complex can be found in the corresponding UV-vis and XANES spectra. In case of the UVvis spectra (Figure 8) the formation of a band at around 430 $\mathrm{nm}$ is observed, which can be assigned to an MLCT transition in the $\mathrm{Cu}^{+}$complex. ${ }^{31}$ Furthermore, the XANES data (Figure 9) show the formation of a shoulder at $8982 \mathrm{eV}$, which gives proof that $\mathrm{Cu}^{+}$is formed under the influence of the X-ray beam.

The snapshots in Figure 7 and the Movie S4 in the Supporting Information also illustrate that, although we use a microreactor in a "stopped-flow" manner, there is some internal sample movement since we observe colored and uncolored areas moving throughout the cuvette. It is most likely that this is caused by heating of the solution by the X-ray beam, which, over the period of time that these measurements were taken, can become significant. ${ }^{4,13}$ However, the reduction process is much quicker than the rate of heat transfer, suggesting that physical heating is not a major contributor to the reduction process. From Figures $3 \mathrm{~b}$ and $7 \mathrm{~b}$ it can clearly be seen that the reduction process occurs rapidly on the right-hand site, where the X-ray beam enters the cuvette. Since most of the X-ray photons are consumed at the entrance of the cuvette, the effect gradually decreases with increasing distance from the entrance.

Factors Influencing the Susceptibility of Copper Complexes to Reduction. In the first part of the paper we have

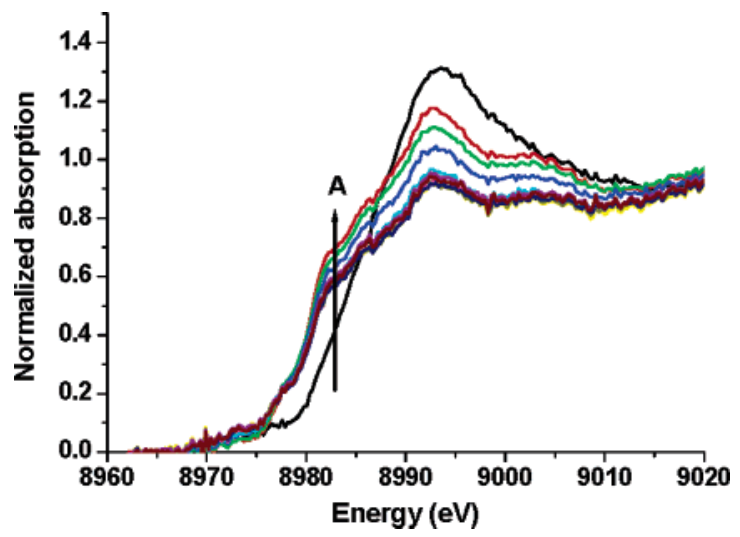

Figure 9. XANES spectra of the copper-neocuproine solution as a function of the reaction time. The arrow labeled A illustrates the growth of a feature at $8982 \mathrm{eV}$. The time interval between two successive XANES spectra is $20 \mathrm{~s}$.

demonstrated the important influence of the X-ray beam on the stability of aqueous copper solutions as a function of time. In this part we will discuss some of the experimental factors that influence copper reduction with regard to the species produced via radiolysis. There are a number of different possible pathways via which the reduction of the $\mathrm{Cu}^{2+}$ cation in the copper complexes may occur. The first two pathways involve the hydrated electron, which has a comparatively high reduction potential $(-2.9 \mathrm{~V})^{34}$ and could either react directly with the $\mathrm{Cu}^{2+}$ or, via a more indirect mechanism, be taken up by the coordinating ligand before being transferred to the $\mathrm{Cu}^{2+}$ cation. The third pathway involves reduction of $\mathrm{Cu}^{2+}$ by radical or ionic species that are subsequently generated by the energetic electrons. These species may be products from the water radiolysis process, but may also be generated as a result of radiation damage to the ligands also present in solution. In all pathways it is clear that the ligands that coordinate to the $\mathrm{Cu}^{2+}$ will have an important role in the degree or rate of reduction. For example, the way in which the ligands coordinate to the $\mathrm{Cu}^{2+}$ species may prevent the hydrated electron or reactive species from reaching $\mathrm{Cu}^{2+}$. However, the ligands might also act as a facilitator for the transport of an electron to $\mathrm{Cu}^{2+}$. Alternatively, they may influence the redox potential, thus making it easier or more difficult for reduction of $\mathrm{Cu}^{2+}$ to occur. Consequently, it is important to consider the ligand when one tries to explain the phenomenon and the observed trends. Therefore, we have selected a number of different ligands and tested them for their susceptibility to reduction under the influence of the X-ray beam in an aqueous solution. A sample of some of the more interesting results is presented in Table S1 in the Supporting Information. The ligands in the table are ordered according to the reduction potential $\left(\mathrm{Cu}^{2+} \mathrm{L}\right.$ to $\left.\mathrm{Cu}^{+} \mathrm{L}\right)$ of the corresponding copper complexes.

A first glance of the data presented in Table S1 suggests that there is no one obvious parameter, which can explain the differences observed in reduction behavior. We therefore discuss our results in relation to the three most likely parameters to have an effect: the reduction potential, the counteranion, and the ligand. First, we consider the significance of the reduction potential. It is clear that there is no correlation between this value and the extent of reduction, suggesting it is not the dominant parameter. For example, on the basis of this parameter alone, in the presence of $100 \mathrm{mM}$ sulfate anion, one would expect the extent of reduction to follow, for three of the ligands, the order ammonium $>$ terpyridine $>$ histidine, when in fact 
reduction is only observed when histidine is the ligand. The apparent lack of correlation in the reduction potential might be due to the fact that it is a measure of $\mathrm{Cu}^{2+} \mathrm{L}_{x}$ to $\mathrm{Cu}^{+} \mathrm{L}_{x}$ reduction rather than colloidal copper formation. However, reduction was observed for the ammonium species in the presence of chloride and bromide anions, which suggests that the counteranion has a particularly important role to play. Reduction of $\mathrm{Cu}^{2+}$ in the presence of chloride and bromide anions, but not in the case of nitrate and sulfate, can be explained by the ability of the nitrate and sulfate to scavenge the hydrated electron, whereas the chloride and bromide are known to be possible $\mathrm{OH}^{\bullet}$ radical scavengers. ${ }^{13,25,35}$ Indeed with the exception of the imidazole ligand, in every case when the $\left[\mathrm{SO}_{4}{ }^{2-}\right]$ is high $(100 \mathrm{mM})$, copper reduction is suppressed. This leads us to conclude that the hydrated electron is most likely to be responsible for reduction of the copper in this solution, but it is not clear whether this occurs via direct interaction with the $\mathrm{Cu}^{2+}$ or via the ligand.

The importance of the ligand can be observed when the results obtained for the neocuproine and terpyridine are compared to all other results. With these ligands only reduction to $\mathrm{Cu}^{+}$or no reduction at all was observed even in the presence of radical or hydrated electron scavengers. [The fact that $\mathrm{Cu}$ (II) to $\mathrm{Cu}(\mathrm{I})$ reduction was observed with neocuproine but not for terpyridine might be due to the more positive electrode potential of the former.] This appears to suggest that the type of ligand and how it coordinates to the copper also plays an important role: the terpyridine ligand, for example, might be able to provide steric protection of the copper cation and prevent it from being reduced by the hydrated electrons, whereas glycine or the ammonia ligand cannot. Indeed, the importance of the ligand coordination behavior might explain the difference in the rate of reduction observed in the copper-histidine samples. It is known for the copper-histidine complex that at $\mathrm{pH} 3.4$ the imidazole ring does not coordinate the copper cation, whereas at $\mathrm{pH} 6.0$ it does. ${ }^{29,30}$ As was shown in the first part, at $\mathrm{pH} 6.0$ the reduction processes occurring were much faster than at $\mathrm{pH}$ 3.4, which could suggest that imidazole coordination of copper enhances the reduction of the copper. However, one could also argue that the presence of either additional $\mathrm{Na}^{+}$ions (added as $\mathrm{NaOH}$ to increase the solution $\mathrm{pH}$ ) or a smaller amount of electron-scavenging $\mathrm{H}_{3} \mathrm{O}^{+}$could be responsible for the enhanced reducing influence seen in the $\mathrm{pH} 6.0$ sample compared to that seen in the $\mathrm{pH} 3.4$ sample. ${ }^{36}$

On the basis of previous work it is possible to make some general observations about a plausible mechanism which leads to the formation of colloidal copper species. First, a partial reduction observed with the $\mathrm{Cu}^{2+}$-neocuproine complex suggests that the first step leading to colloidal copper formation is a reduction from $\mathrm{Cu}^{2+}$ to $\mathrm{Cu}^{+}$, eq 2 . The comparatively high reduction potential meant that this was readily achieved but that the stability of $\mathrm{Cu}^{+}-$neocuproine complexes with respect to $\mathrm{Cu}^{0}$ or reoxidation to $\mathrm{Cu}^{2+}$ is more difficult to achieve with this complex than with other complexes. ${ }^{37}$ However, the question of colloidal cluster formation is more difficult to rationalize. It is clear that for dimeric and larger copper clusters to form the copper complexes would need to be in close proximity to each other. However, whether complexation occurs via the reaction between two reduced $\mathrm{Cu}^{0}$-containing complexes (eq 3) or between a $\mathrm{Cu}^{0}$-containing complex and $\mathrm{a} \mathrm{Cu}^{+}$containing complex before coalescence and dispropotionation takes place is not clear (eq 4). ${ }^{34}$ However, it is possible, using the spectroscopic data, to effectively rule out the formation of nanosized $\mathrm{Cu}_{2} \mathrm{O}$ as a possible intermediate leading to colloidal copper formation. ${ }^{32}$

$$
\begin{gathered}
\mathrm{Cu}^{\mathrm{II}} \mathrm{L}_{x}+\mathrm{e}_{(\text {aq })}^{-} \rightarrow \mathrm{Cu}^{\mathrm{I} \mathrm{L}_{\mathrm{x}}} \\
2 \mathrm{Cu}^{0} \mathrm{~L}_{x} \rightarrow \mathrm{Cu}_{2}+2 \mathrm{~L}_{x} \rightarrow x \mathrm{Cu}_{2}+\mathrm{Cu}_{2 x} \\
\mathrm{Cu}^{\mathrm{I}} \mathrm{L}_{x}+\mathrm{Cu}^{0} \mathrm{~L}_{x} \rightarrow \mathrm{Cu}_{2}^{+}+2 \mathrm{~L}_{x}
\end{gathered}
$$

\section{Conclusions}

In this paper we have demonstrated the benefits of using a combined spectroscopic approach to observe the effects that $\mathrm{X}$-rays may have on aqueous solutions containing transitionmetal ions. Under the influence of the X-rays radiolysis of water instantaneously occurred, which resulted in the production of reactive species, which have a big reducing influence on the solutes that are present in the solution. These results suggested that the hydrated electron was responsible for reduction, although it was not clear whether this was via a direct interaction with $\mathrm{Cu}^{2+}$ or through an initial interaction with the coordinating ligand. However, the extent of metal ion reduction did show a dependency on the ligand type (we have considered mainly the coordination behavior rather than the ability of the ligand to scavenge hydrated electrons), the nature of the counteranion, and possibly the reduction potential. On the basis of these conclusions, one could argue whether it is possible to study catalytic mechanisms in water-containing systems by using X-ray absorption spectroscopy, as the application of the spectroscopic technique introduces in situ new, very reactive species within the catalytic system. Of course, the phenomena occurring are stimulated in our case, as a highly focused highflux X-ray beam is used. However, the same radiolysis products are generated at low X-ray fluxes and at less focused beamlines, although the effects might be much less visible.

The results also show the benefits of using combined in situ spectroscopic techniques to observe and understand the processes that occur during in situ reaction and, in particular, to probe the influence that one technique (particularly those that employ ionizing radiation) has on the sample stability. In addition, we have demonstrated the benefits of using real time "video spectroscopy" for the understanding of the phenomena that occur in the volume of the cuvette.

Acknowledgment. The ESRF (Grenoble, France) is acknowledged for the provision of its facilities. Virginie Belliere, Pieter Bruinincx, and Kaisa Kervinen (all from Utrecht University (UU)) and Gemma Guilera, Mark Newton, and Florian Perrin (from ID24, ESRF) are thanked for their help and useful discussions during the ED-XANES experiments. Jan den Boesterd (UU) is acknowledged for digitizing the video movies. This research work has been sponsored by an NWO-CW VICI grant to B.M.W.

Supporting Information Available: Experimental details on the copper complexes tested for their susceptibility to reduction under the influence of the X-ray beam and movies showing the effect of these irradiation processes. This material is available free of charge via the Internet at http://pubs.acs.org.

\section{References and Notes}

(1) Teo, B. K. EXANES: Basic Principles and Data Analysis; SpringerVerlag: New York, 1986.

(2) Koningsberger, D. C., Prins, R., Eds. X-ray Absorption, principles, applications, techniques of EXANES, SEXANES and XANES; WileyInterscience: New York, 1988.

(3) Timneanu, N.; Caleman, C.; Hadju, J.; van der Spoel, D. J. Chem. Phys. 2004, 299, 277.

(4) Teng, T.; Moffat, K. J. Synchrotron Radiat. 2000, 7, 313. 
(5) Cherezov, V.; Cheng, A.; Petit, J. M.; Diat, O.; Caffrey, M. Cell. Mol. Biol. 2000, 46, 1133.

(6) Nave, C.; Hill, M. A. J. Synchrotron Radiat. 2005, 12, 299.

(7) Döllefeld, H.; McGinley, C.; Almousalami, S.; Möller, T.; Weller, H.; Eychmüller, A. J. Chem. Phys. 2002, 117, 8953.

(8) Andreas, B.; Breunig, I.; Buse, K. ChemPhysChem 2005, 6, 1544.

(9) Menou, N.; Castagnos, A. M.; Muller, C.; Goguenheim, D.; Goux,

L.; Wouters, D. J.; Hodeau, J. L.; Dooryhee, E.; Barrett, R. J. Appl. Phys. 2005, 97, 044106.

(10) O’Neill, P.; Stevens, D. L.; Garman, E. F. J. Synchrotron Radiat. 2002, 9, 329.

(11) Sommerhalter, M.; Lieberman, R. L.; Rosenzweig, A. C. Inorg. Chem. 2005, 44, 770 .

(12) Bergmann, U.; Glatzel, P.; Cramer, S. P. Microchem. J. 2002, 71, 221.

(13) Mesu, J. G.; van der Eerden, A. M. J.; de Groot, F. M. F.; Weckhuysen, B. M. J. Phys. Chem. B 2005, 109, 4042.

(14) Murphy, L. M.; Dobson, B. R.; Neu, M.; Ramsdale, C. R.; Strange,

R. W.; Hasnain, S. S. J. Synchrotron Radiat. 1995, 2, 64.

(15) Zhang, K.; Liu, R. F.; Irving, T.; Auld, D. S. J. Synchrotron Radiat. 2004, 11, 204

(16) Meitzner, G.; Gardea-Toffesdey, J.; Parsons, J.; Scott, S. L.; Deguns, E. W. Microchem. J. 2005, 81, 61.

(17) Murray, J.; Garman E. F. J. Synchrotron Radiat. 2002, 9, 347.

(18) Jayanetti, S.; Mayanovic, R. A.; Anderson, A. J.; Bassett, W. A.; Chou, I. M. J. Chem. Phys. 2001, 115, 954.

(19) Lee, H. J.; Je J. H.; Hwu, Y.; Tsai W. L. Nucl. Instrum. Methods Phys. Res., B 2003, 199, 342.

(20) Ma, Q.; Moldovan, N.; Mancini, D. C.; Rosenburg, R. A. Appl. Phys. Lett. 2000, 76, 2014.
(21) Tromp, M. Developments of Time-Resolved XANES Spectroscopy Techniques. Ph.D. Thesis, Utrecht University, 2004.

(22) Stein, G., Ed. Radiation Chemistry of Aqueous Systems; The Weizmann Science Press of Israel: Jerusalem, 1968.

(23) Allen, A. O. The Radiation Chemistry of Water and Aqueous Solutions; D. van Nostrand Co., Inc.: Princeton, NJ, 1961.

(24) Maleknia, S. D.; Brenowitz, M.; Chance, M. R. Anal. Chem. 1999, 71,3965 .

(25) Pastina, B.; LaVerne, J. A.; Pimblott, S. M. J. Phys. Chem. A 1999, 103,5841

(26) LaVerne, J. A.; Pimblott, S. M. J. Phys. Chem. A 2000, 104, 9820.

(27) Garrett, B. C.; et al. Chem. Rev. 2005, 105, 355.

(28) Gamez, P.; Arends, I. W. C. E.; Sheldon, R. A.; Reedijk, J. Adv. Synth. Catal. 2004, 346, 805 .

(29) Mesu, J. G. Host-Guest Chemistry of $\mathrm{Cu}^{2+} /$ Histidine Complexes in Molecular Sieves. Ph.D. Thesis, Utrecht University, 2005.

(30) Mesu, J. G.; Visser, T.; Soulimani, F.; van Faassen, E. E.; de Peinder, P.; Beale, A. M.; Weckhuysen, B. M. Inorg. Chem. 2006, 45, 1960.

(31) Parker, W. L.; Crosby, G. A. J. Phys. Chem. 1989, 93, 5692.

(32) Deki, S.; Akamatsu, K.; Yano, T.; Mizuhata, M.; Kajinami, A. J. Mater. Chem. 1998, 8, 1865.

(33) Wu, S. H.; Chen, D. H. J. Colloid Interface Sci. 2004, 273, 165.

(34) Henglein, A. J. Phys. Chem. B 2000, 104, 1206.

(35) Pimblott, S. M. J. Phys. Chem. 1992, 96, 4485.

(36) Boyle, J. W.; Ghormley, J. A.; Hochanadel, C. J.; Riley, J. F. J. Phys. Chem. 1969, 73, 2886.

(37) Sözgen, K.; Demirci Cekic, S.; Tütem, E.; Apak, R. Talanta 2006, 68,1601 